Society Awards 1995

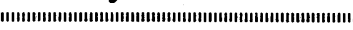

(on high prospectiveness)

\title{
Mechanism of Action of $N$-Phenylimide Photobleaching Herbicides
}

\author{
Ryo Sato \\ Agricultural Chemicals Research Laboratory, Sumitomo Chemical Co., Ltd., \\ Takatsukasa, Takarazuka 665, Japan
}

Light is involved in the action of many types of herbicides, but its role may not be primary. Diphenyl ethers (DPE) such as acifluorfenethyl (AFE) do not show any visible herbicidal symptoms in darkness. Because of their distinct light-dependent mode of action, characteristic herbicidal symptoms including rapid desiccation, bleaching, browning, necrosis in treated plants, they are often called photobleaching herbicides. However this conclusion is reached without knowledge of the metabolic process with which DPEs interfere. An experimental $N$-phenylimide herbicide S23142 ( $N$-[4-chloro-2-fluoro-5-propargyloxyphenyl]-3,4,5,6-tetrahydrophthalimide) has the typical mode of action of photobleaching herbicides, despite a chemical structure of its completely different from the DPEs.

In order to investigate the mechanism of action of S-23142 and related photobleaching herbicides, the physiological and biochemical effects of S-23142 and AFE were studied. Unlike diuron, bromoxynil and paraquat, S23142 and AFE neither inhibited the Hill reaction nor enhanced oxygen uptake in isolated chloroplast fragments. In excised cucumber cotyledons, S-23142, as well as AFE, enhanced ethane production and caused leakage of cytosolic substances including ATP and amino acids into the medium. This suggests that S-23142 and AFE have a common mechanism of action that causes membrane damage by enhancing peroxidation of membrane lipids. Massive accumulation of porphyrins, loss of photosynthesis, and ethylene production under stress were also observed in cucum- ber cotyledons treated with S-23142 and AFE. Porphyrin accumulation has been reported to be caused by many other photobleaching herbicides as well and could be regarded as the most characteristic action of these herbicides.

To clarify the photoreceptor pigment(s) involved in the action of S-23142 and AFE, the wavelengths of light effective in inducing herbicidal action in cucumber cotyledons were determined. In green tissue, activities of S-23142 and AFE showed identical wavelength effects with peaks at 450,550 , and $650 \mathrm{~nm}$, which indicates involvement of common photoreceptor(s) in the action of these herbicides. Since the wavelength effects suggested that chlorophyll-related pigments might be the photoreceptor(s), similar experiments were conducted using chlorophyll depleted tissue and S-23142. In etiolated tissue grown in far-red light, near UV light at $400 \mathrm{~nm}$ was as effective for induction of S-23142 activity as in green tissue, while blue $(450 \mathrm{~nm})$ and red $(650$ $\mathrm{nm})$ light were much less effective than in green tissue. In tentoxin-treated yellow cotyledons, the wavelength effect on the activity of S-23142 showed a single peak at $400 \mathrm{~nm}$ and is distinct from that observed in green tissue. These results strongly suggested that multiple photoreceptors (photoreactions) are involved in the herbicidal action of S-23142 and that a chlorophyll-related pigment is the possible photoreceptor in green tissue but not in chlorophyll-depleted tissue. Since the wavelength effect on S-23142 activity in tentoxintreated yellow tissue matches absorption spectra of porphyrins, these pigments are to 
be involved in the action of photobleaching herbicides in both green and yellow tissue.

In cucumber cotyledons treated with S23142, porphyrin accumulation is observed within $1 \mathrm{hr}$ after treatment even in darkness, suggesting a close connection to the primary action of the photobleaching herbicides. At least four porphyrins including protoporphyrin IX (Proto) were separated from S-23142 treated cucumber cotyledons by HPLC. Time course analysis of porphyrin content in etiolated cucumber cotyledons transferred to light after S-23142 treatment showed that accumulation of Proto was accompanied by a reduction in chlorophyll and Mg-Proto amounts, indicating that $\mathrm{Mg}$-Proto formation is inhibited in vivo. However, S-23142 did not inhibit Mg-chelatase activity in greening etioplasts of cucumber cotyledons. In contrast, S-23142 did inhibit protoporphyrinogen oxidase (Protox) activity from greening radish seedlings more strongly than did AFE at very low concentrations $\left(10^{-9} \mathrm{M}\right)$. From these results, I conclude that Protox is the biochemical site of action of S-23412 as well as other photobleaching herbicides, supporting the idea first proposed by Matringe et al. (FEBS Lett. 245, 35 (1989)) that, in the presence of herbicide, protoporphyrinogen (Protogen) may be exported from the porphyrin pathway and oxidized to Proto at abnormal site(s).

Since accumulated porphyrins in homogenates of herbicide treated tissue separate with the membrane fraction during centrifugation, the possibility that Proto may form a complex with a membrane bound polypeptide was tested. Membrane proteins from cucumber cotyledons exposed to S-23142 were solubilized with $1 \% n$-dodecyl- $\beta$-D-maltoside $(\mathrm{DM})$, and separated by polyacrylamide gel electrophoresis containing $0.2 \%$ digitonin (DGPAGE) at 4C. Some fluorescent porphyrins migrated with a membrane polypeptide, forming a porphyrin-protein complex. The porphyrin-protein complex was also detected by gel permeation HPLC separation of solubilized membrane proteins. The molecular mass of the polypeptide was estimated to be $63 \mathrm{kDa}$ by SDS-PAGE. The $63 \mathrm{kDa}$ protein was also found in untreated cotyledons in the absence of porphyrin binding. A $63 \mathrm{kDa}$ porphyrin-protein complex was also formed by mixing authentic Proto and solubilized membrane proteins prepared from untreated cotyledons in vitro. Under these conditions, Proto bound to none of the other membrane proteins nor to soluble proteins or BSA, indicating that the $63 \mathrm{kDa}$ protein has a very high affinity for Proto. Since Proto is hydrophobic and is known to accumulate in membrane systems in cells treated with photobleaching herbicides, the porphyrin-protein complex may also form in vivo and be involved in the action of photobleaching herbicides.

To identify the primary site of action of S23142 , the receptor protein of S-23142 must be identified. For this purpose, binding of ${ }^{14} \mathrm{C}$ S-23121, an S-23142 analog, to solubilized plastid proteins of corn seedlings was studied. Membrane proteins were solubilized with $1 \%$ $\mathrm{DM}$, incubated with ${ }^{14} \mathrm{C}-\mathrm{S}-23121$, and trapped by polyethyleneimine coated glass filters to estimate the bound radioactivity. Scatchard analysis of the binding curve indicated the presence of a single binding site and its dissociation constant ( $c a .9 \mathrm{~nm}$ ) was consistent with inhibitory activity of S-23121 on Proto synthesizing enzyme activity. S-23142 and AFE competed with ${ }^{14} \mathrm{C}-\mathrm{S}-23121$ in binding to this receptor, while DCMU did not. These results suggested that the S-23121 binding protein is highly likely to be the receptor protein for photobleaching herbicides.

To investigate the mechanism of action of S-23142 at the molecular level, S-23142 resistant mutants of unicellular green alga Chlamydomonas reinhardtii have been isolated, since $C$. reinhardtii shows essentially the same highly sensitive responses to photobleaching herbicides as higher plants. One of these resistant mutants, $r s-3$, possessed the highest resistance level to S-23142 and tolerates up to ca. 100 times higher concentration of S-23142 than the wild type strain CC-407 in terms of growth or porphyrin accumulation. This mutant was also cross-resistant to other photobleaching herbicides including oxadiazon, oxyfluorfen, and AFE but showed the wild type level of sensitivity to herbicides with different modes of action. This suggests that a common site of action of photobleaching herbicides may be mutated to acquire resistance. Genetic 
analysis of the $r s-3$ mutation revealed that resistance results from a single dominant nuclear mutation that maps to linkage group IX, 13.7 map units from $s r-1$ (streptomycin resistance). Protox activity in Percoll-purified chloroplast fragments prepared from $r s-3$ was less sensitive to S-23142 and AFE than Protox activity from wild type, indicating that $r s-3$ mutation confers resistance at the enzyme level. Since no significant difference was found in the amounts of Protox activity per chlorophyll or protein between the mutant and wild type, the enzyme resistance may be due to reduced binding of herbicide by the enzyme.

To identify the $r s-3$ gene, a cosmid library of $r s-3$ nuclear DNA has been constructed. 10,080 Escherichia coli clones has been frozen individually as an indexed library and an unordered library consisting of a mixture of more than 10,000 clones has also been prepared. Since $r s-3$ is a dominant nuclear mutation, efforts have been made to isolate S-23142 resistant cells by transforming arginine requiring, cell wall deficient CC-425 (arg-2, $c w-15)$ strain with total $r s-3$ library DNA, and one S-23142 resistant isolate has been obtained. Although no cosmid vector sequence was found by Southern analysis, the isolate may still be a true transformant, since it shows a very similar cross resistance profile to $r s-3$ and has the resistance allele present at a different locus from the $r s-3$ allele. Vector sequences are not always integrated into the genome in nuclear transformants of Chlamydomonas, especially in the rare transformants that occur by homologous gene replacement. Since no S-23142 resistant isolates were seen in control experiments, this result suggests that $r s-3$ gene can be isolated by screening the genomic library. Isolation and identification of $r s-3$ gene will lead to further understanding of the molecular mechanism of action of photobleaching herbicides. 